\title{
Differential Consequences of Bmp9 Deletion on Sinusoidal Endothelial Cell Differentiation and Liver Fibrosis in 129/Ola and C57BL/6 Mice
}

\author{
Agnès Desroches-Castan, Emmanuelle Tillet ${ }^{\circledR}$, Nicolas Ricard ${ }^{\dagger}$, Marie Ouarné ${ }^{\ddagger}$, \\ Christine Mallet, Jean-Jacques Feige and Sabine Bailly * \\ Biology of Cancer and Infection Laboratory, University Grenoble Alpes, Inserm, CEA, F-38000 Grenoble, France; \\ agnes.castan@cea.fr (A.D.-C.); emmanuelle.tillet@cea.fr (E.T.); nic.ricard@gmail.com (N.R.); \\ marie.ouarne@medicina.ulisboa.pt (M.O.); christine.mallet@cea.fr (C.M.); jean-jacques.feige@cea.fr (J.-J.F.) \\ * Correspondence: sabine.bailly@cea.fr; Tel.: +(33)-43-878-9214 \\ † Present address: Yale Cardiovascular Research Center, Yale University School of Medicine, New Haven, \\ CT 06511, USA. \\ ‡ Present address: Instituto de Medicina Molecular, Faculdade de Medicina, Universidade de \\ Lisboa,1649-028 Lisbon, Portugal.
}

Received: 29 July 2019; Accepted: 10 September 2019; Published: 13 September 2019

\begin{abstract}
The aim of the present work was to address the role of BMP9 in different genetic backgrounds (C57BL/6, BALB/c, and 129/Ola) of mice deleted for Bmp9. We found that Bmp9 deletion led to premature mortality only in the 129/Ola strain. We have previously shown that Bmp9 deletion led to liver sinusoidal endothelial cells (LSEC) capillarization and liver fibrosis in the 129/Ola background. Here, we showed that this is not the case in the C57BL/6 background. Analysis of LSEC from Wild-type (WT) versus Bmp9-KO mice in the C57BL/6 background showed no difference in LSEC fenestration and in the expression of differentiation markers. Comparison of the mRNA expression of LSEC differentiation markers between WT C57BL/6 and 129/Ola mice showed a significant decrease in Stabilin2, Plvap, and CD209b, suggesting a more capillary-like phenotype in WT C57BL/6 LSECs. C57BL/6 mice also had lower BMP9 circulating concentrations and hepatic Vegfr2 mRNA levels, compared to the 129/Ola mice. Taken together, our observations support a role for BMP9 in liver endothelial cell fenestration and prevention of fibrosis that is dependent on genetic background. It also suggests that 129/Ola mice are a more suitable model than C57BL/6 for the study of liver fibrosis subsequent to LSEC capillarization.
\end{abstract}

Keywords: BMP9; genetic background; liver; fibrosis; capillarization; liver sinusoidal endothelial cells; HHT; mouse; fenestrae; plvap

\section{Introduction}

BMP9 (Bone morphogenetic protein 9), also known as GDF2 (growth and differentiation factor 2), belongs to the TGF $\beta$ family whose members are involved in many different biological activities $[1,2]$. BMP9 is produced by hepatic stellate cells (HSCs) [3,4]. BMP9 is present in blood at active concentrations and is now recognized to be a potent vascular quiescence factor [4,5]. Recombinant BMP9 was also shown to be one of the most potent osteogenic inducers and a regulator of several biological functions, including chondrogenesis, glucose metabolism, neuronal differentiation, and iron balance [6]. BMP9 is the physiological high affinity ligand (Kd: $2 \mathrm{pM}$ ) of the endothelial-specific receptor ALK1 (activin receptor-like kinase 1) and of its co-receptor endoglin [7-9].

The role of BMP9 in liver homeostasis is still unclear and controversial [10,11]. BMP9 was reported to be an autocrine/paracrine cytokine inhibiting adult (non-malignant) hepatocyte proliferation [3]. 
Recently, Breitkopf-Heinlein et al. and Addante et al. published that BMP9 is a profibrogenic factor in the liver [3,12]. In line with these works, Li et al. found that higher BMP9 levels accompanied advanced stages of liver fibrosis [13]. In contrast, analysis of distinct transcriptomic datasets from those mentioned above revealed that hepatic BMP9 mRNA expression is decreased in several human liver diseases including cirrhosis, NASH (non-alcoholic steato hepatitis) and hepatocarcinomas [14]. We have recently addressed the role of BMP9 in liver homeostasis by studying the phenotype of Bmp9 deleted mice in the 129/Ola genetic background [15]. In this previous work, we found that Bmp9 deletion triggers hepatic perisinusoidal fibrosis by regulating the differentiated state of liver sinusoidal endothelial cells (LSEC), leading to a dedifferentiated state of LSEC. This dedifferentiation state is called capillarization and corresponds to the loss of LSEC-specific markers, loss of LSEC fenestration, and formation of an organized basement membrane in the space of Disse [16]. Capillarization often precedes human liver fibrosis [17].

In humans, heterozygous mutations of either ACVRL1 (encoding ALK1) or ENG (encoding Endoglin, an endothelial co-receptor for MP9/10) genes cause hereditary hemorrhagic telangiectasia (HHT), a genetic disease with multisystemic vascular defects ranging from small cutaneous and mucosal telangiectasias, to severe arteriovenous malformations (AVMs) in the lung, liver, and the brain, which drive severe cardiac complications $[18,19]$. HHT presents significant phenotypic variability, wherein the age of symptoms onset, the location of telangiectasias and AVMs, and the severity of the disease vary widely between individuals carrying the same mutation, even within the same family. These significant intra-familial differences support a role for genetic modifiers [20]. Indeed, the PTPN14gene, encoding the non-receptor tyrosine phosphatase 14, was identified as a potential modifier gene whose polymorphisms influence the severity of pulmonary arteriovenous malformations [21]. Heterozygous mice for Acorl1 or Eng reproduce some HHT-like lesions but with a low frequency [22]. Interestingly, these HHT-like lesions have been shown to be more frequent in the 129/Ola than in the C57BL/6 genetic background, suggesting again that genetic modifiers might play a role in susceptibility to the HHT disease $[23,24]$.

These data prompted us to evaluate the phenotype of Bmp9-KO mice under distinct genetic backgrounds, namely C57BL/6, 129/Ola, and BALB/c. We found large differences between these different mouse strains. Bmp9 deletion leads to premature mortality only in the 129/Ola genetic background. Analysis of the livers of C57BL/6 versus 129/Ola mice in both WT and Bmp9-KO animals showed that loss of Bmp9 in the C57BL/6 strain did not lead to liver fibrosis nor to LSEC capillarization. Our data further suggest differences in the status of the LSEC-differentiated state between these two mouse strains.

\section{Materials and Methods}

\subsection{Animal Experimentations}

Institutional guidelines elaborated by the European Community for the Use of Experimental Animals were followed for all animal experiments (agreement APAFIS\#9436-2017032916298306) the approval of the CEA ethics committee and the French Ministry of Research and Education were received. We obtained Bmp9-KO mice in the C57BL/6 genetic background from Dr. Se-Jin Lee (Johns Hopkins University, Baltimore, MD). These mice were previously characterized [25] and back-crossed for 10 generations with either 129-P2/OlaHsd wild-type (WT) mice [26] (Harlan/Envigo, Gannat, France) or BALB/c WT mice (Charles River, Ecully, France) in order to obtain Bmp9-KO mice from these two genetic backgrounds. The 129-P2/OlaHsd WT mice are hereafter called 129/Ola for the sake of simplification. These mice were bred under a specific-pathogen-free (SPF) animal facility. We rapidly noticed that the Bmp9 deletion in the 129/Ola background lead to a damageable phenotype with premature mortality-one week before death, these mice presented an important weight loss $(10-20 \%$ of their initial weight). Subsequently, the mice were weighted once a week and euthanized as soon they had lost $20 \%$ of their weight. 


\subsection{Histological and Immunohistochemical Procedures}

Livers were harvested from carbon dioxide-euthanized animals. Organs were fixed by overnight immersion in $4 \%$ formaldehyde and then embedded in paraffin after progressive alcoholic dehydration steps. Hematoxylin-eosin and Sirius-red stainings were performed on $5-\mu \mathrm{m}$-thick deparaffinized sections (all products from Sigma-Aldrich, St. Louis, MO, USA). The fibrotic response was defined as the ratio between the Sirius-red-positive areas and the total surface of the tissue section. For Collagen IV immunohistochemistry, deparaffinized sections were incubated overnight at room temperature with a primary antibody (Abcam ab19808), after Tris-EDTA buffer antigen retrieval protocol. Appropriate secondary antibody was subsequently incubated for $1 \mathrm{~h}$ at room temperature. All microscopy pictures were taken using a Zeiss Axioplan microscope (Zeiss, Oberkochen, Germany) and analyses and quantifications were performed using the Axiovision 4.9.1 image analysis software (Zeiss). Briefly, for the sinusoid area quantification, white areas corresponding to the vessels including veins, sinusoids, and arteries were automatically detected and then veins, arteries and bile ducts were manually removed and the area of the sinusoids were calculated as the percentage of the entire picture area.

\subsection{Liver Sinusoid Endothelial Cells (LSEC) Isolation and Culture}

These cells were isolated as previously described [26]. In brief, we perfused livers from anesthetized mice (26-30-weeks-old females) via the inferior veina cava with warm EGTA buffer (while the portal vein was sectioned), and subsequently by warm collagenase II (Merck-Biochrom, Berlin, Germany). Livers were harvested and mechanically dissociated. Cells were isolated by centrifugation through Histodenz (Sigma-Aldrich) gradients.

Freshly isolated LSECs were immediately used (before cell culture) for mRNA expression studies.

For scanning electron microscopy (SEM), LSECs were seeded on fibronectin-coated coverslips and grown in EGM-2 supplemented with 10\% FCS for 4 to $6 \mathrm{~h}$ under a $19 \% \mathrm{O}_{2}$ and $5 \% \mathrm{CO}_{2}$ atmosphere. This condition was considered as optimal for cell spreading and fenestration observation.

\subsection{Scanning Electron Microscopy (SEM)}

LSECs were fixed overnight with $2 \%$ glutaraldehyde in $0.125 \mathrm{M}$ phosphate buffer $\mathrm{pH} 7.2$. They were then post-fixed for $1 \mathrm{~h}$ with $1 \%$ osmium tetroxide $\left(\mathrm{OsO}_{4}\right)$, dehydrated with graded ethanol and desiccated by a $30 \mathrm{~min}$ immersion in hexamethyldisilazane followed by air drying. Samples were copper-metallized and observed on a Quanta 250 FEG scanning electron microscope at the "Centre Technologique des Microstructures" of University Claude Bernard (Lyon, France). For each condition, fenestrae were quantified on 86 to 105 images, using the multi-point tool of the ImageJ software.

\subsection{RNA Extraction and Quantitative RT-PCR}

Fresh livers were homogenized in RNA later (Sigma-Aldrich). RNA extraction was carried out from 80-100 mg of tissue using Trizol (Thermo Fisher Scientific, Carlsbad, CA, USA), according to the manufacturer's instructions. For freshly isolated LSECs, RNA was extracted using the RNA-XS Plus extraction kit (Macherey-Nagel, Düren, Germany). A total of $1 \mu \mathrm{g}$ of RNA was reverse-transcribed using the iScript kit (Biorad, Hercules, CA, USA). Quantitative RT-PCR experiments were performed using the SYBR-green master-mix GoTaq (Promega, Charbonnières-les-Bains, France) on a CFX96 thermocycler (Biorad). mRNA expression levels were normalized to the level of either VE-cadherin (Cdh5) or Rpl13a, as indicated by the figure legends. All primers are detailed in the supplementary information Table S1. The qPCR data were calculated using the $\Delta \Delta \mathrm{Ct}$ method or the $\Delta \mathrm{Ct}$ method as specified in the figure legends. 


\subsection{Enzyme-Linked Immunosorbent Assay (ELISA)}

Hyaluronan levels from mouse plasma (40-weeks-old females) were quantified according to the manufacturer's recommendation (DHYALO Biotechne, Minneapolis, MN, USA).

BMP9 ELISA measurement has been previously described [4]. MAB3209 (R\&D Systems) antibody for BMP9 was used as capture antibody and detection was performed using biotinylated antibody (anti mature BMP9: BAF3209, R\&D Systems). Plasma of 12 (129/Ola WT) and 12 (C57BL/6) mice were quantified.

\subsection{BMP Activity Measurement}

NIH-3T3 cells were transfected with a mixture of the reporter plasmid pGL3(BRE)2-luc encoding firefly luciferase, downstream of a BMP response element, pRL-TK luc encoding Renilla luciferase and a plasmid encoding human ALK1, as previously described [7].

\subsection{Statistical Analysis}

Data were analyzed using the Prism6 software (GraphPad, La Jolla, CA, USA). The non-parametric tests (Mann-Whitney or Kruskal-Wallis) used are indicated in the figure legends. Results were considered to be statistically significant when $p$-value $<0.05$.

\section{Results}

\subsection{Bmp9 Deletion Leads to Premature Mortality in a Gender-and Strain-Dependent Manner}

Bmp9 genetic deletion was initially generated in C57BL/6 mice by the team of Dr. S.J. Lee. These mice were viable and fertile and exhibited no overt defect under SPF husbandry conditions, except for lymphatic vessels dilation and lymphatic valves malformations [25,27]. To address whether Bmp9 deletion could lead to different phenotypes depending on the genetic background, these mice were intercrossed for 10 generations with wild-type 129/Ola or BALB/c mice, in order to generate Bmp9-KO mice under these two additional genetic backgrounds. We then analyzed their overall survival at the adult stage. In agreement with others and our previous work $[25,28]$, there was no effect of Bmp9 deletion on the overall survival in the C57BL/6 genetic background (Figure 1A). The overall survival of Bmp9-KO mice in the BALB/c strain was also not different from that of WT mice (Figure 1B). In contrast, Bmp9 deletion in the 129/Ola strain led to premature death (Figure 1C). Interestingly, we observed a clear difference between the males and the females. Males died earlier (mean survival age of 28 weeks) than females (mean survival age of 49 weeks) (Figure 1C). Careful analysis of the different organs of these mice at autopsy revealed macroscopic defects in two main organs-livers had patchy white spots on their surface, as previously described [15] and sometimes hyperdilated vessels. Kidneys also displayed patchy white spots, some were hemorrhagic and sometimes translucid. Kidneys were affected in $40 \%$ of the males versus $4 \%$ of the females while the liver was affected in both males (22\%) and females (45\%) (Figure 1D). We noticed that death of males was often preceded by a severe weight-loss (10-20\% loss over the week preceding death) (data not shown), suggesting that the defects in the kidney function could be the cause of male death. This early mortality in males explains in part the lower percentage of liver defects in males as they die before developing liver defects. Taken together, these data show a clear difference in the overall survival of Bmp9-KO mice, depending on gender and strain. 
A

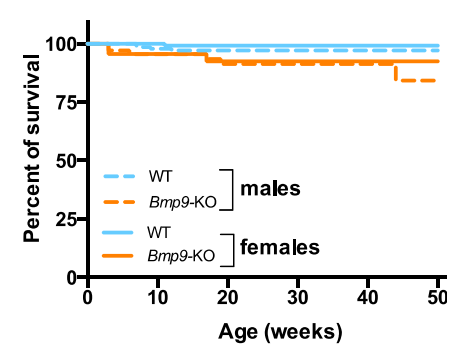

D

129/Ola

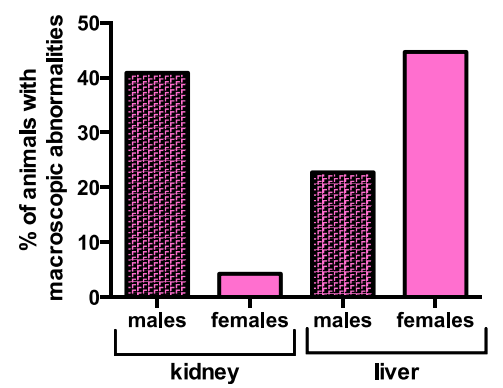

B

BALB/c

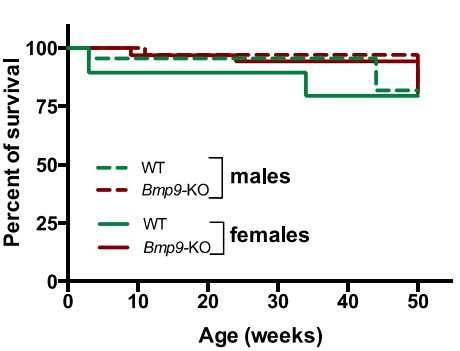

C

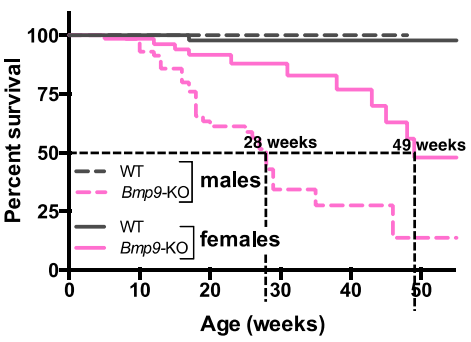

Figure 1. Survival curves of Bmp9-KO mice of three genetic backgrounds. (A) Survival in C57BL/6 background (wild-type (WT) males $n=154$, WT females $n=168, B m p 9-\mathrm{KO}$ males $n=63$, Bmp9-KO females $n=43$ ). (B) Survival in BALB/c background (WT males $n=42$, WT females $n=49$, Bmp9-KO males $n=44$, Bmp9-KO females $n=75$ ). (C) Survival in 129/Ola background (WT males $n=62$, WT females $n=61, B m p 9-\mathrm{KO}$ males $n=44, B m p 9-\mathrm{KO}$ females $n=59$ ). (D) Macroscopic alterations of kidneys and the liver revealed at autopsy in 129/Ola Bmp9-KO mice before 50 weeks of age. Results are expressed in terms of percentage of total autopsied animals (females $n=51$; males $n=61$ ).

\subsection{Bmp9 Deletion in the C57Bl/6 Strain Does not Lead to Liver Fibrosis}

We have recently shown that $B m p 9$ deletion in the 129/Ola genetic background leads to spontaneous liver fibrosis [15]. We thus wanted to know if $B m p 9$ deletion under other genetic backgrounds could also lead to liver fibrosis. We focused on the C57BL/6 genetic background which is the main background studied in gene KO experiments. For this, liver sections of adult WT and Bmp9-KO mice from 129/Ola and C57BL/6 strains were stained with Sirius red. We found a significant increase in Sirius-red-stained collagen deposits in 129/Ola Bmp9-KO livers (Figure 2A,B) but none in the C57BL/6 Bmp9-KO livers. Occasionally, we noted few red spots on the liver sections of the C57BL/6 Bmp9-KO mice. We have also previously shown that liver sections from 129/Ola Bmp9-KO mice presented dilated sinusoidal vessels [15]. We thus stained liver sections with Hematoxylin-Eosin and measured the surface of sinusoidal vessels in each condition, excluding veins and arteries from this quantification. We found no difference in the sinusoidal size between WT and Bmp 9 -KO mice from the C57BL/6 strain (Figure 2C,D) whereas, the sinusoids were significantly dilated in Bmp 9 -KO livers from the 129/Ola mice, as previously shown [15]. Together, these data showed that Bmp9 deletion in the C57BL/6 background did not lead to liver fibrosis nor to sinusoidal vessel enlargement. 

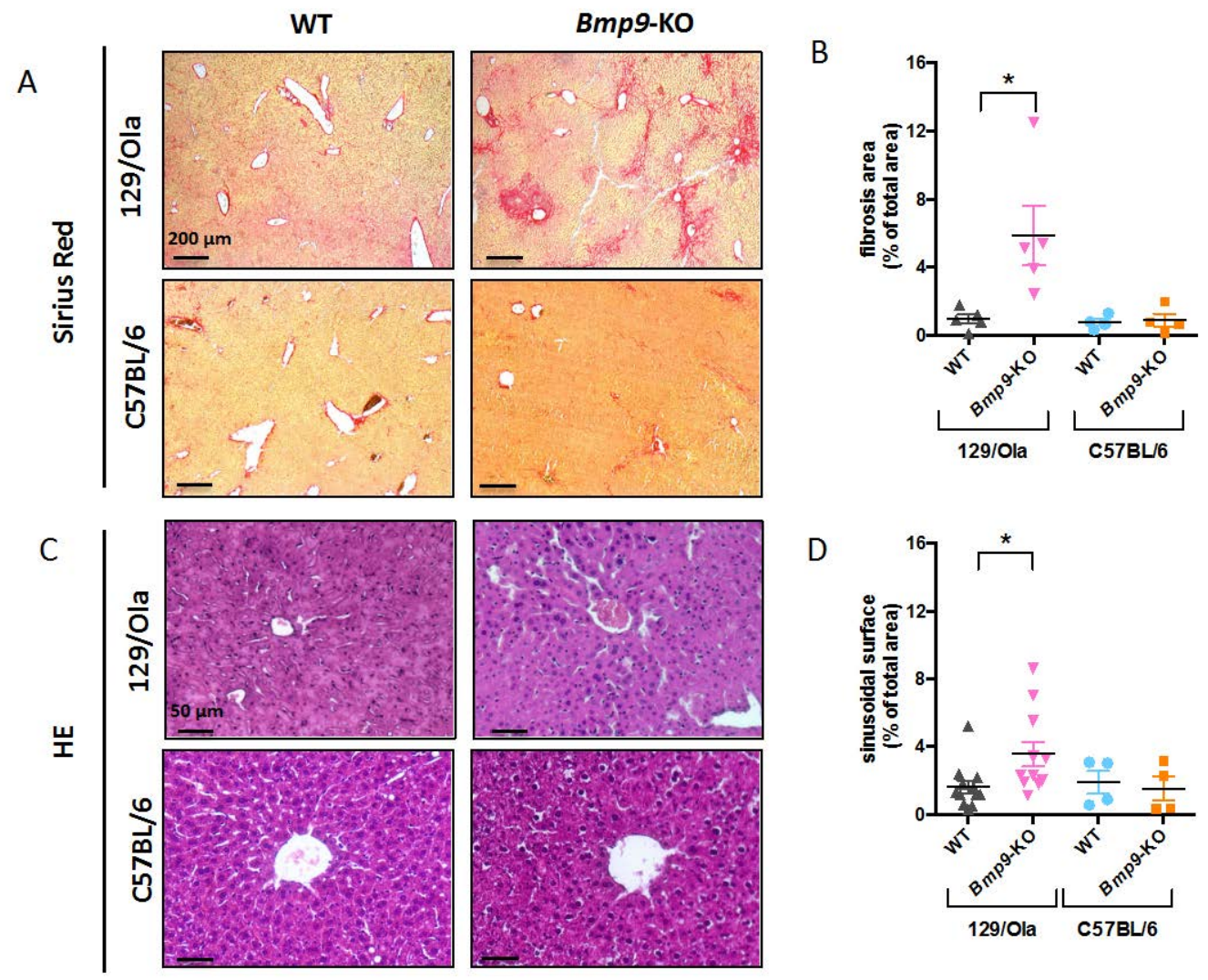

Figure 2. $\mathrm{C} 57 \mathrm{Bl} / 6$ and 129/Ola Bmp9-KO mice display different liver phenotypes. (A) Sirius red histochemical staining of the liver sections $(5 \mu \mathrm{m})$ of WT and Bmp9-KO mice from the 129/Ola and C57BL/6 strain. (B) Quantification of fibrotic surface in percentage of total area ( $n=4$ in each group, 5 images analyzed per mouse). Fibrotic areas appear in red and were quantified using the Axiovision software. (C) Hematoxylin and Eosin histochemical staining of liver sections $(5 \mu \mathrm{m})$ from WT and Bmp9-KO mice from 129/Ola and C57BL/6 strain. (D) Quantification of sinusoidal surface measured using Axiovision software and expressed as percentage of total area $(n=4-12$ mice per group, 5 images analyzed per mouse). Central veins and arteries were excluded from this quantification. Data are expressed as mean \pm standard error of the mean (SEM) of duplicate determinations from multiple samples. The Kruskal-Wallis test was used for all statistical analysis of this figure. ${ }^{*} p<0.05$.

\subsection{Bmp9 Deletion in the C57Bl/6 Strain Does Not Modify Liver Sinusoidal Endothelial Cell (LSEC) Differentiation State}

We have previously shown that Bmp9 deletion led to liver fibrosis, which was preceded by capillarization of LSEC [15]. We thus analyzed these different parameters in the LSEC isolated from adult livers of WT versus Bmp9-KO mice from the C57BL/6 strain. We first analyzed LSEC fenestration by scanning electron microscopy (SEM) after LSEC plating and culture for $18 \mathrm{~h}$, as previously described [15]. Surprisingly, we found a very small number of fenestrae in the WT LSEC from the C57BL/6 strain (data not shown), compared to the WT LSEC from the 129/Ola strain [15]. As LSEC fenestration decreases rapidly in cells during in vitro culture [29], we looked at an earlier time point that was after $6 \mathrm{~h}$ of culture. Again, we observed a very low density of fenestrae $\left(<0.5\right.$ fenestrae $/ \mu \mathrm{m}^{2}$; Figure 3A) as compared to the number of fenestrae from the WT LSEC derived from 129/Ola mice under the same culture conditions ( $>5$ fenestrae $/ \mu \mathrm{m}^{2}$; Figure S1). When we compared the LSEC fenestration from WT to the Bmp9-KO mice in the C57BL/6 strain, we found no significant difference (Figure 3A). Several specific markers of the LSEC terminal differentiation were previously described by Geraud et al. [30], including Gata4 and Maf, two key hepatic transcription factors, Plvap (plasmalemmal vesicle-associated protein; it encodes a constitutive fenestra protein), Stabilin-1 and Stabilin-2 encoding the scavenger receptors, Ehd3 encoding an endocytic receptor, and Cd209b encoding an adhesion 
molecule. The mRNAs of these different markers were found to be significantly decreased in the 129/Ola Bmp9-KO mice, as compared to the WT mice [15]. We thus analyzed the expression of these markers in the C57BL/6 LSECs from the WT and Bmp9-KO mice. The expression of these genes was evaluated by RT-qPCR. As shown in Figure 3B, none of these mRNAs was differentially expressed between the Bmp9-KO and WT mice. Another marker of endothelial capillarization is the deposition of a basal lamina around sinusoidal endothelial cells, which is normally absent in a normal liver [31]. We thus stained the liver sections from WT and Bmp9-KO mice with an antibody against Collagen IV, which is one of the main constituents of basal lamina. We observed a comparable level of Collagen IV staining (Figure 3C) in the WT and the Bmp9-KO mice from the C57BL/6 strain. This result was different to the one observed in the 129/Ola background. In this background, the level of collagen IV was very low in the WT mice and high in the Bmp9-KO (Figure S1C), in accordance with our previous work [15]. We also assessed Collagen4a4 mRNA expression by RT-qPCR and found similar levels between the WT and the Bmp9-KO mice (Figure 3D). Finally, we measured circulating hyaluronan levels, which reflected the activity of the scavenger receptor Stabilin-2, in the plasma of these mice. No difference in the HA circulating levels was found between the WT and the Bmp9-KO mice (Figure 3E). Taken together, these results showed no difference between the LSEC from the WT and the Bmp9-KO in the C57BL/6 background. It clearly appeared, however, that the LSEC from the WT C57BL/6 mice presented a capillarized phenotype, with a low density of fenestrae and the presence of a basal lamina, along the sinusoids.

A

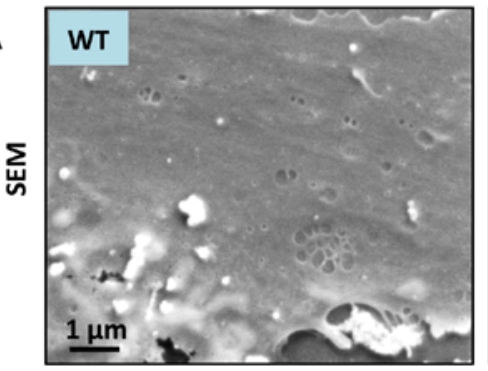

B
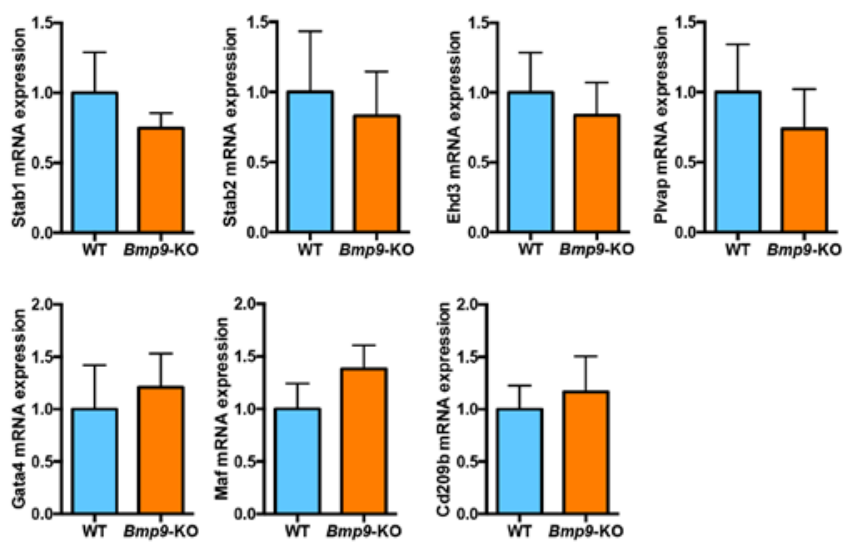

C
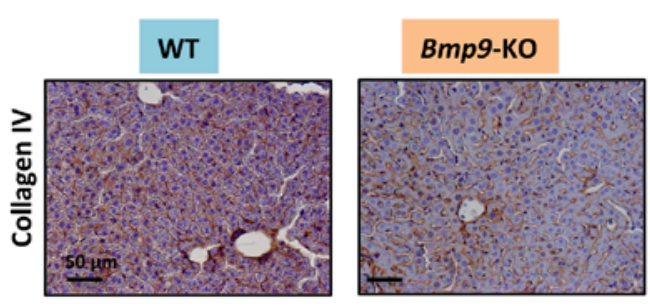

D

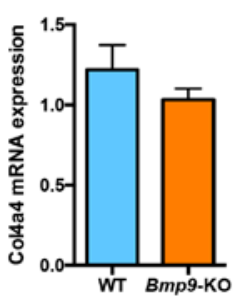

$\mathrm{E}$

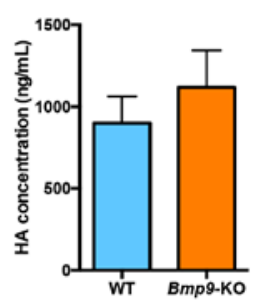

Figure 3. Liver sinusoidal endothelial cells (LSEC) capillarization in WT and Bmp9-KO mice in the C57BL/6 genetic background. (A) Representative pictures of Scanning Electron Microscopy (SEM) on 
freshly isolated LSEC from C57BL/6 mice (26-weeks-old females), 4-6 h after plating. Graph on the right shows the quantification of fenestrae per $\mu \mathrm{m}^{2}$ assessed using the ImageJ software $(n=5$ for WT mice, 86 images quantified; and $n=4$ for Bmp9-KO mice, 105 images quantified). (B) mRNA expression levels of the LSEC markers measured by the RT-qPCR ( $n=5$ and 4 for respectively WT and Bmp9-KO mice). mRNA expression levels were normalized to Vascular Endothelial Cadherin (VE-Cadherin) (Cdh5). The results are expressed as a ratio to the WT mean value ( $\Delta \Delta \mathrm{Ct}$ method). (C) Representative images of Collagen IV immunohistochemical staining on liver sections $(5 \mu \mathrm{m})$ from WT and Bmp9-KO mice in the C57BL/6 genetic background ( $n=4$ in each group). (D) mRNA expression of Col4a 4 in livers from WT and Bmp9-KO mice in the C57BL/6 genetic background ( $n=6$ in each group). Values were normalized to Rpl13a mRNA expression levels. The results are expressed as a ratio to the WT mean value. (E) Circulating levels of hyaluronan (HA) were measured by ELISA in at least 6 plasma samples from both WT and Bmp9-KO mice of the C57Bl/6 genetic background. Data are plotted as mean $\pm(\mathrm{SEM})$. All data were statically analyzed with the Mann-Whitney test. No significant difference was observed ( $p$-values $>0.05)$.

\subsection{C57BL/6 and 129/Ola LSEC from WT Mice Express Different Levels of Hepatic Endothelial Terminal Differentiation Markers}

We next compared the LSEC terminal differentiation markers between the WT mice from the C57BL/6 and the 129/Ola strains. Interestingly, we found a significant difference in the mRNA levels of Stabilin-2, Plvap, and Cd209b, which were significantly lower in the C57BL/6 WT mice, as compared to the 129/Ola WT mice (Figure 4). The same trend, although it did not reach statistical significance, could also be observed in the expression of the two transcription factors Gata4 and Maf. On the other hand, the mRNA levels of Stab1, Edh3, and eNOS were not different between the two strains. VE-Cadherin mRNA levels were not different between the two strains, supporting the idea that we analyzed the same amount of LSEC mRNA between the two strains. Taken together, these data indicate again that C57BL/6 LSEC express a less terminally differentiated and a more "capillary-like" phenotype than the 129/Ola LSEC.
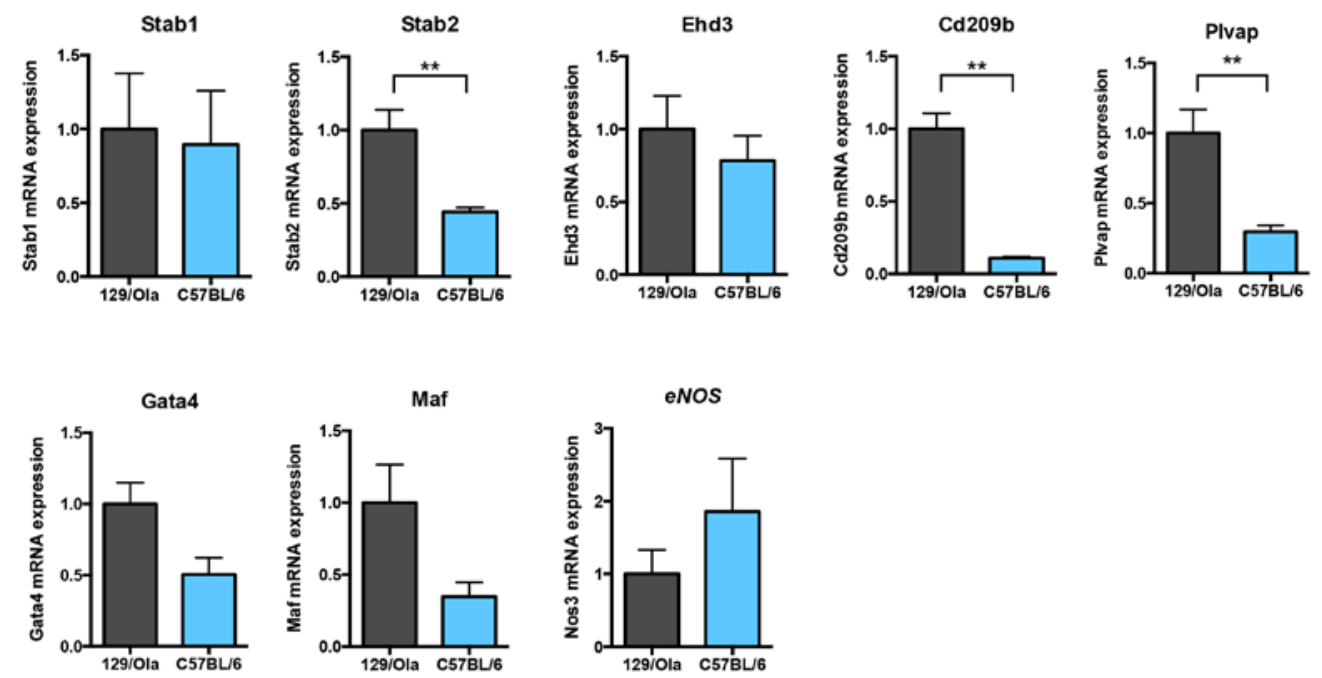

Figure 4. mRNA levels of LSEC differentiation markers in the WT mice of 129/Ola and C57BL/6 genetic backgrounds. mRNA expression levels of the LSEC markers were analyzed by RT-qPCR from freshly prepared LSEC from six 129/Ola and four C57BL/6 WT mice. Values were normalized to Rpl13a mRNA expression levels and the results are expressed as a ratio of these values to that of $129 / \mathrm{Ola}(\Delta \Delta \mathrm{Ct}$ method). Data are plotted as mean \pm SEM. Statistical analysis was performed using the Mann-Whitney test. ${ }^{* *} p<0.01$. 


\subsection{C57BL/6 WT Mice Have Lower Levels of Circulating BMP9 and BMP-Activity than 129/Ola Mice}

We then investigated the BMP9/ALK1 signaling pathway first by RT-qPCR on freshly prepared LSECS from the C57BL/6 and 129/Ola strains. No difference in the expression of the BMP9 receptors (ALK1, ALK2, ALK5, BMPR2, ActR2A, ENG) could be found between the two strains (Figure 5A). mRNA analysis of the different BMP-specific SMADs (Smad1, Smad5, and Smad9) again showed no difference between the two strains. However, there was a significant decrease in C57BL/6 LSEC versus 129/Ola of Smad6 mRNA levels and a slight decrease of the Id1 mRNA levels, which are the two main BMP9 target genes (Figure 5A). Smad7 mRNA levels were not different between the two strains. We next measured the plasmatic BMP9 levels by ELISA in the two strains. We found a significantly lower level of circulating BMP9 in the WT C57BL/6 mice versus the 129/Ola mice (Figure 5B). This decrease was confirmed when we measured the BMP-ALK1-dependent activity from these plasma, using a BRE-luciferase reporter assay (Figure 5C).
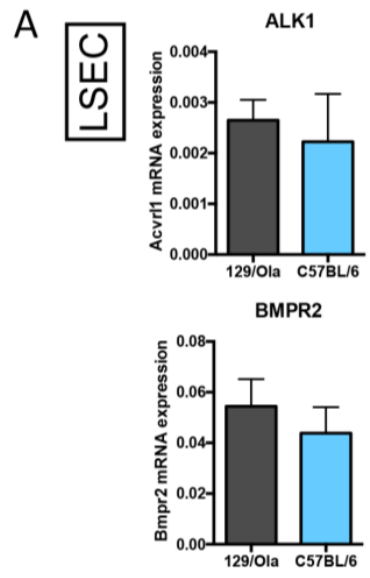

Smad1
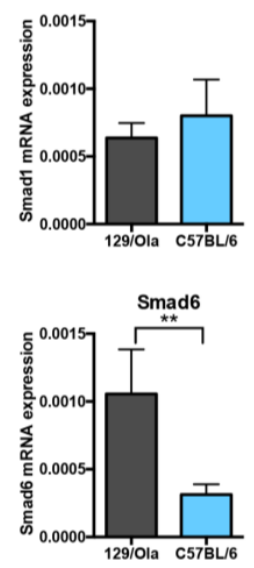
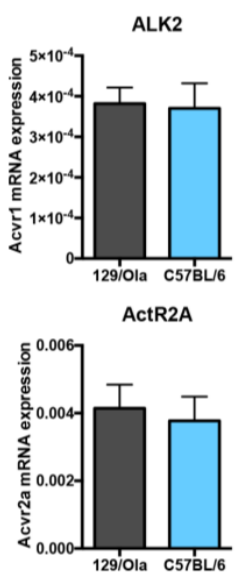

Smad5
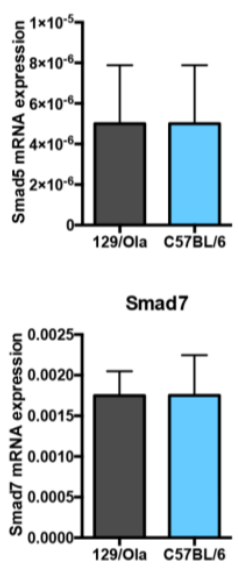

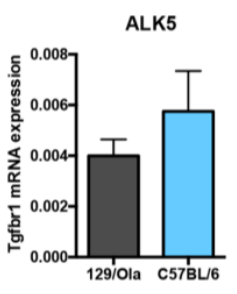

ENG

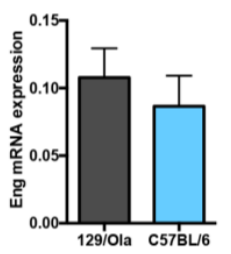

Smad9

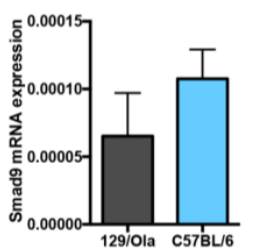

Id1

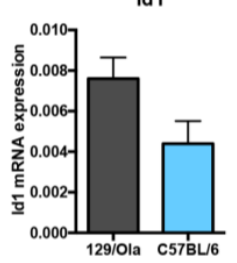

B
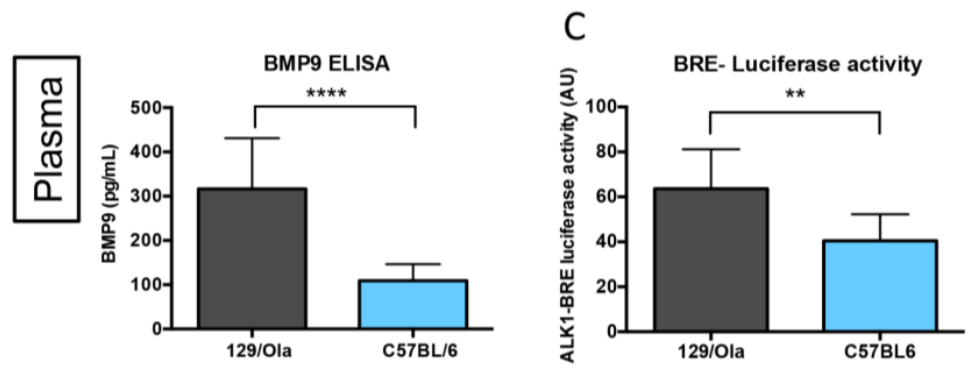

Figure 5. BMP9/ALK1 signaling pathway in 129/Ola and the C57BL/6 WT mice. (A) Quantification of the mRNA expression level of type I (ALK1, ALK2, ALK5) and type II (BMPR2, ActR2A) receptors, the 
coreceptor endoglin, the transcription factors (Smad1, Smad5 and Smad9), and the target genes (Smad6, Smad7 and Id1), from freshly prepared LSEC of six 129/Ola and four C57BL/6 mice. Expression was normalized to the Rpl13a mRNA expression ( $\triangle \mathrm{Ct}$ method). (B) BMP9 plasma levels measured by ELISA in the 129/Ola and the C57BL/6 WT mice (males, 6 to 8 months old, $n=12$ in each group). (C) ALK1-BRE luciferase activity (AU: Arbitrary Units) in plasma from 129/Ola and C57BL/6 WT mice (6-8-months-old males, $n=12$ in each group). Data are plotted as mean \pm SEM. Statistical analysis was performed using the Mann-Whitney test. ${ }^{* *} p<0.01$; ${ }^{* * * *} p<0.0001$.

\subsection{C57BL/6 WT Mice Express Lower Vegfr2 mRNA Levels than 129/Ola Mice}

We then investigated whether the Vascular Endothelial Growth Factor (VEGF) signaling pathway which has been previously described as a key player in the LSEC capillarization [29,32], was different in the liver of WT C57BL/6 mice versus that of WT 129/Ola mice. Vegfa mRNA levels were not different between the two strains (Figure 6). However, we found that Kdr (VEGFR2) mRNA levels were significantly decreased in C57BL/6 as compared to the 129/Ola mice, while the mRNA levels of the other receptors of this pathway Flt1 (VEGFR1) and Flt4 (VEGFR3) were not different between the two strains (Figure 6).
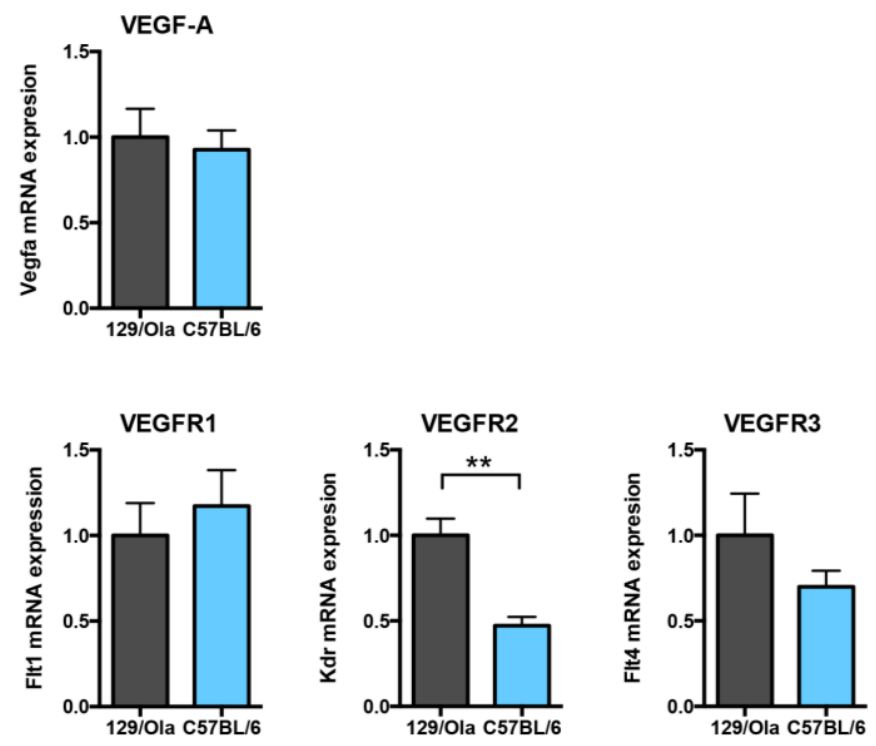

Figure 6. mRNA levels of the Vascular Endothelial Growth Factor (VEGF) signaling pathway in the livers of WT mice of 129/Ola and C57BL/6 genetic backgrounds. Quantification of the mRNA expression level of Vegfa (VEGF-A) and its three receptors Vegfr2, Flt1 (VEGFR1), and Flt4 (VEGFR3) from the livers of the 129/Ola and the C57BL/ 6 mice ( $n=4-5$ and $n=6$, respectively). Values are normalized to the Rpl13a mRNA expression levels and the results are expressed as a ratio of these values to that of $129 /$ Ola $(\Delta \Delta \mathrm{Ct}$ method). Data are plotted as mean $\pm \mathrm{SEM}$. Statistical analysis was performed using the Mann-Whitney test. ${ }^{* *} p<0.01$.

\section{Discussion}

BMP9 is a growth factor, produced by hepatic stellate cells that is found in the circulation under biological active concentrations [3,4]. It has been reported to act on the vascular endothelium to maintain vessel quiescence via its high affinity endothelial-specific receptor ALK1 [7,9]. We have recently reported that BMP9 plays a central role in liver homeostasis as genetic deletion of Bmp9 in the 129/Ola mice led to spontaneous hepatic fibrosis [15]. Here, we showed that this effect is strain-dependent, as Bmp9 deletion in the C57BL/6 strain does not lead to spontaneous liver fibrosis. Comparing the LSEC status in the two different genetic backgrounds showed a differential state of sinusoidal endothelial differentiation. Indeed, C57BL/6-derived LSEC presented several characteristics that suggested a less differentiated and "capillary-like" phenotype, as compared to the 129/Ola LSEC. 
The already capillary-like phenotype of LSEC derived from the C57BL/6 mice might explain why Bmp 9 deletion, which we previously showed to induce LSEC capillarization [15], did not lead to a more pronounced capillarized phenotype.

One original observation of the present work is that the phenotypic alterations induced by genetic deletion of Gdf2/Bmp 9 in mice vary as a function of the genetic background of the inbred mouse strain used. We report here that the 129/Ola strain is highly sensitive to Bmp9 gene deletion, leading to premature death during adulthood. In contrast, C57BL/6 and BALB/c Bmp9-KO mice have a normal life expectancy. It has been long known that many biological responses vary from one strain of inbred mice to another and in particular the angiogenic response [33]. In particular, 129/Ola mice have been shown to be more sensitive to vascular stimuli than the C57BL/6 mice [34]. Difference between mouse strains has also been shown to affect genetic regulation of the liver gene expression network [35]. This could result from genomic differences as the deep sequencing of the genomes of 36 commonly used inbred mouse strains by the Wellcome Trust Sanger Institute, recently allowed to identify more than 800 protein-encoding genes with the premature stop codons reducing the length of the proteins by more than $20 \%$ [36]. This work, together with our recently published study, clearly supports a role for BMP9 in liver homeostasis, which depends on the genetic background and supports the notion of genetic modifiers in general and more particularly in HHT $[20,21]$. Our work further supports that studying the function of a protein should not be limited to one mouse line, as one could miss the function of this protein.

In the present work, we identified major differences in the state of mouse LSEC differentiation between C57BL/6 and 129/Ola strains. Collagen IV staining of liver sections of C57BL/6 mice showed the presence of a basal lamina that was not present in the 129/Ola strain (Figure 3C and Figure S1C). Analysis of freshly isolated LSEC from these two strains also showed a lower density of fenestrae in the C57BL/6 mice versus the 129/Ola (Figure 3A and Figure S1C). Furthermore, consistent with these results, several markers that have been specifically identified in LSEC [30] were differentially expressed (Stabilin2, Plvap, and Cd209b) between these two mouse strains. Plvap is a dimeric protein constituent of the diaphragm of diaphragmed-fenestrae (present in kidney peritubular capillaries, capillaries of intestinal villi, pancreas, adrenal cortex, endocrine glands, and choriocapillaries of the brain and eye) [37] but it has also been shown to be required for the formation of non-diaphragmed fenestrae, such as those present in LSECs [38]. Interestingly, in line with our results, it has been recently shown by two different groups that deletion of the Pvlap gene leads to phenotypes that depend on the genetic background [39,40]. On pure C57BL/6J background, homozygous Plvap deletion resulted in 100\% embryonic lethality, while in a mixed background some pups were born and survived for up to 4 weeks. Stab1 $1^{--}$-Stab2 ${ }^{-/}$mice have been generated in two different backgrounds and it was shown that these double KO mice on a C57BL/6 background showed only moderately elevated levels of albumin in the urine, in comparison to the BALB/c background [41]. These data, together with our results, demonstrated that fenestrae formation and expression of terminal differentiation markers are dependent on the genetic background. Interestingly, we found a significantly lower level of active circulating BMP9 in the plasma of C57BL/6 mice versus that of the 129/Ola mice, which could, at least in part, explain the differences of the differentiation states between these two strains, as we have previously demonstrated that BMP9 is a key factor for LSEC maturation [15]. Interestingly, we also found that livers from the C57BL/6 expressed a lower level of Kdr (VEGFR2) mRNA levels than the 129/Ola mice. Maintenance of the LSEC differentiation was shown to require VEGF-A [29,32]. Thus, a lower level of the VEGFR2, the main VEGF-A receptor, could also explain the "capillarized" state of the WT LSEC from the C57BL/6 versus that of the 129/Ola mice.

Our data suggest a different state of the LSEC differentiation between these two mouse strains. C57BL/6 LSEC seem to have a more "capillary-like" phenotype than the 129/Ola LSEC. Capillarization has been reported to precede liver fibrosis in human patients and animal models [17,31], thus, one would expect to see more spontaneous liver fibrosis in the C57BL/6 mice. However, we did not observe liver fibrosis in the WT C57BL/6 mice. It is, thus, likely that the C57BL/6 mice have developed a 
physiological adaptation to this peculiar LSEC phenotype. In particular, since LSEC fenestrae appear to facilitate the uptake of chylomicron remnants from the portal circulation, it remains to be understood whether the remaining number of fenestrae is sufficient for lipid uptake or if C57BL/6 mice have developed alternative mechanisms to control lipidemia.

The absence of spontaneous liver fibrosis in Bmp9-KO mice in the C57BL/6 background is compatible with the work of Breitkopf-Heinlein et al., [3]. In their work, they proposed that BMP9 stabilizes hepatocyte function in healthy livers but that BMP9 could become harmful under conditions of liver damage. These opposites functions of BMP9 have also been observed in malignant versus non-malignant liver conditions [42,43], supporting the context-dependent role of BMP9. It will be interesting in the future to challenge Bmp9-KO mice of the 129/Ola background, which spontaneously develop liver fibrosis under conditions of liver damage. In the present work, we have only addressed the role of BMP9 on LSEC, but it has been demonstrated in previous studies that BMP9 can modulate HSC and hepatocyte activities $[3,11]$. It will, thus, be important to study the finely tuned crosstalk between the different liver cell types driven by BMP9 to better understand the role of this key factor in liver homeostasis.

The more differentiated LSEC phenotype observed in the 129/Ola mice might explain why this mouse strain is more prone to liver fibrosis in the absence of Bmp9. This could in part explain, why it is so difficult to develop liver fibrosis models in mice as most studies are performed in C57BL/6 mice [44]. Together, our results suggest that the 129/Ola strain seems to be a good model to study liver fibrosis mediated by capillarization. It has been clearly demonstrated that capillarization precedes the onset of alcoholic liver disease in humans [45]. It would, thus, be interesting in the future to challenge the Bmp9-KO mice of the 129/Ola genetic background with a high-fat diet or to test whether these mice could develop cirrhosis and hepatocarcinoma.

Supplementary Materials: The following are available online at http://www.mdpi.com/2073-4409/8/9/1079/s1, Figure S1: Loss of LSEC fenestration in Bmp9-KO mice of the 129/Ola background, Table S1: List of primers.

Author Contributions: A.D.-C. conceived the work, designed, and performed most of the experiments; analyzed the data; and wrote the manuscript. E.T. performed the experiments and critically revised the manuscript. N.R., M.O., and C.M. performed some experiments and revised the manuscript. J.-J.F. conceived the study; coordinated the work; and revised the manuscript for important intellectual content. S.B. conceived the study; coordinated the work; and drafted and finalized the manuscript. All authors read and approved the final manuscript.

Funding: This research was funded by the Institut National de la Santé et de la Recherche Médicale (INSERM, U1036), the Commissariat à l'Energie Atomique et aux Energies Alternatives (CEA, DRF/IRIG), the University Grenoble Alpes (UGA, BCI), the Fondation pour la Recherche Médicale (FRM), the Association Maladie de Rendu-Osler (AMRO/HHT-France), the Association pour la Recherche sur le Cancer (ARC), the Ligues Départementales contre le Cancer de l'Isère et de la Savoie, the Agence Nationale de la Recherche (ANR grant no. ANR-17-CE14-0006; Be9inPAH), and the Plan Cancer Inserm 2014-2019 OncoSWIR.

Acknowledgments: We are indebted to Hervé Pointu, Nicolas Chaumontel, Soumalamaya Bama, and Irène Jeannin for their help in the maintenance of our mouse colonies. The authors acknowledge the animal facility platform supported by GRAL, financed within the University Grenoble Alpes graduate school (Ecoles Universitaires de Recherche) CBH-EUR-GS (ANR-17-EURE-0003). We thank Cécile Maës and Alexia Giroud for their contributions to some of these experiments.

Conflicts of Interest: The authors declare no conflict of interest.

\section{References}

1. Tillet, E.; Bailly, S. Emerging roles of bmp9 and bmp10 in hereditary hemorrhagic telangiectasia. Front. Genet. 2014, 5, 456. [CrossRef] [PubMed]

2. Wood, J.H.; Guo, J.; Morrell, N.W.; Li, W. Advances in the molecular regulation of endothelial BMP9 signalling complexes and implications for cardiovascular disease. Biochem. Soc. Trans. 2019, 47, 779-791. [CrossRef] [PubMed]

3. Breitkopf-Heinlein, K.; Meyer, C.; König, C.; Gaitantzi, H.; Addante, A.; Thomas, M.; Wiercinska, E.; Cai, C.; Li, Q.; Wan, F.; et al. BMP-9 interferes with liver regeneration and promotes liver fibrosis. Gut 2017, 66, 939-954. [CrossRef] [PubMed] 
4. Tillet, E.; Ouarné, M.; Desroches-Castan, A.; Mallet, C.; Subileau, M.; Didier, R.; Lioutsko, A.; Belthier, G.; Feige, J.-J.; Bailly, S. A heterodimer formed by bone morphogenetic protein 9 (BMP9) and BMP10 provides most BMP biological activity in plasma. J. Biol. Chem. 2018, 293, 10963-10974. [CrossRef] [PubMed]

5. David, L.; Mallet, C.; Keramidas, M.; Lamandé, N.; Gasc, J.-M.; Dupuis-Girod, S.; Plauchu, H.; Feige, J.-J.; Bailly, S. Bone morphogenetic protein-9 is a circulating vascular quiescence factor. Circ. Res. 2008, 102,914-922. [CrossRef] [PubMed]

6. Leblanc, E.; Drouin, G.; Grenier, G.; Faucheux, N.; Hamdy, R. From skeletal to non skeletal: The intriguing roles of bmp-9: A litterature review. Adv. Biosci. Biotechnol. 2013, 4, 31-46. [CrossRef]

7. David, L.; Mallet, C.; Mazerbourg, S.; Feige, J.-J.; Bailly, S. Identification of BMP9 and BMP10 as functional activators of the orphan activin receptor-like kinase 1 (ALK1) in endothelial cells. Blood 2007, 109, $1953-1961$. [CrossRef] [PubMed]

8. $\quad$ Brown, M.A.; Zhao, Q.; Baker, K.A.; Naik, C.; Chen, C.; Pukac, L.; Singh, M.; Tsareva, T.; Parice, Y.; Mahoney, A.; et al. Crytal structure of bmp-9 and functional interaction with pro-region and receptors. J. Biol. Chem. 2005, 280, 25111-25118. [CrossRef]

9. Scharpfenecker, M.; Van Dinther, M.; Liu, Z.; Van Bezooijen, R.; Zhao, Q.; Pukac, L.; Löwik, C.W.G.M.; Dijke, P.T. BMP-9 signals via ALK1 and inhibits bFGF-induced endothelial cell proliferation and VEGF-stimulated angiogenesis. J. Cell Sci. 2007, 120, 964-972. [CrossRef]

10. Herrera, B.; Addante, A.; Sanchez, A. Bmp signalling at the crossroad of liver fibrosis and regeneration. Int. J. Mol. Sci. 2018, 19, 39. [CrossRef]

11. Herrera, B.; Dooley, S.; Breitkopf-Heinlein, K. Potential Roles of Bone Morphogenetic Protein (BMP)-9 in Human Liver Diseases. Int. J. Mol. Sci. 2014, 15, 5199-5220. [CrossRef] [PubMed]

12. Addante, A.; Roncero, C.; Almalé, L.; Lazcanoiturburu, N.; García-Álvaro, M.; Fernandez, M.; Sanz, J.; Hammad, S.; Nwosu, Z.C.; Lee, S.-J.; et al. Bone morphogenetic protein 9 as a key regulator of liver progenitor cells in DDC-induced cholestatic liver injury. Liver Int. 2018, 38, 1664-1675. [CrossRef] [PubMed]

13. Li, P.; Li, Y.; Zhu, L.; Yang, Z.; He, J.; Wang, L.; Shang, Q.; Pan, H.; Wang, H.; Ma, X.; et al. Targeting secreted cytokine BMP9 gates the attenuation of hepatic fibrosis. Biochim. Biophys. Acta 2018, 1864, 709-720. [CrossRef] [PubMed]

14. John, M.; Kim, K.J.; Bae, S.D.W.; Qiao, L.; George, J. Role of BMP-9 in human liver disease. Gut 2018, 0, 1-3. [CrossRef] [PubMed]

15. Desroches-Castan, A.; Tillet, E.; Ricard, N.; Ouarné, M.; Mallet, C.; Belmudes, L.; Couté, Y.; Boillot, O.; Scoazec, J.; Bailly, S.; et al. Bone Morphogenetic Protein 9 Is a Paracrine Factor Controlling Liver Sinusoidal Endothelial Cell Fenestration and Protecting Against Hepatic Fibrosis. Hepatology 2019, (in press). [CrossRef] [PubMed]

16. Schaffner, F.; Popper, H. Capillarization of Hepatic Sinusoids in Man. Gastroenterology 1963, 44, $239-242$. [CrossRef]

17. Urashima, S.; Tsutsumi, M.; Nakase, K.; Wang, J.S.; Takada, A. Studies on Capillarization of the Hepatic Sinusoids in Alcoholic Liver Disease. Alcohol Alcohol. 1993, 28, 77-84. [CrossRef]

18. Bailly, S.; Plauchu, H.; Dupuis-Girod, S.; Dupuis-Girod, S.; Dupuis-Girod, S. Hereditary hemorrhagic telangiectasia: From molecular biology to patient care. J. Thromb. Haemost. 2010, 8, 1447-1456.

19. Shovlin, C.L. Hereditary haemorrhagic telangiectasia: Pathophysiology, diagnosis and treatment. Blood Rev. 2010, 24, 203-219. [CrossRef]

20. McDonald, J.; Wooderchak-Donahue, W.; Webb, C.V.; Whitehead, K.; Stevenson, D.A.; Bayrak-Toydemir, P. Hereditary hemorrhagic telangiectasia: Genetics and molecular diagnostics in a new era. Front. Genet. 2015, 6, 1. [CrossRef]

21. Benzinou, M.; Clermont, F.F.; Letteboer, T.G.; Kim, J.H.; Espejel, S.; Harradine, K.A.; Arbelaez, J.; Luu, M.T.; Roy, R.; Quigley, D.; et al. Mouse and human strategies identify ptpn14 as a modifier of angiogenesis and hereditary haemorrhagic telangiectasia. Nature Commun. 2012, 3, 616. [CrossRef] [PubMed]

22. Tual-Chalot, S.; Oh, S.P.; Arthur, H.M. Mouse models of hereditary hemorrhagic telangiectasia: Recent advances and future challenges. Front. Genet. 2015, 6, 25. [CrossRef] [PubMed]

23. Bourdeau, A.; Faughnan, M.E.; McDonald, M.L.; Paterson, A.D.; Wanless, I.R.; Letarte, M. Potential role of modifier genes influencing transforming growth factor-beta1 levels in the development of vascular defects in endoglin heterozygous mice with hereditary hemorrhagic telangiectasia. Am. J. Pathol. 2001, 158, 2011-2020. [CrossRef] 
24. Torsney, E.; Charlton, R.; Diamond, A.G.; Burn, J.; Soames, J.V.; Arthur, H.M. Mouse Model for Hereditary Hemorrhagic Telangiectasia Has a Generalized Vascular Abnormality. Circulation 2003, 107, $1653-1657$. [CrossRef] [PubMed]

25. Ricard, N.; Ciais, D.; Levet, S.; Subileau, M.; Mallet, C.; Zimmers, T.A.; Lee, S.J.; Bidart, M.; Feige, J.-J.; Bailly, S. BMP9 and BMP10 are critical for postnatal retinal vascular remodeling. Blood 2012, 119, 6162-6171. [CrossRef]

26. Prudent, R.; Vassal-Stermann, É.; Nguyen, C.H.; Mollaret, M.; Viallet, J.; Desroches-Castan, A.; Martinez, A.; Barette, C.; Pillet, C.; Valdameri, G.; et al. Azaindole derivatives are inhibitors of microtubule dynamics, with anti-cancer and anti-angiogenic activities. Br. J. Pharmacol. 2013, 168, 673-685. [CrossRef]

27. Levet, S.; Ciais, D.; Merdzhanova, G.; Mallet, C.; Zimmers, T.A.; Lee, S.-J.; Navarro, F.P.; Texier, I.; Feige, J.J.; Bailly, S.; et al. Bone morphogenetic protein 9 (BMP9) controls lymphatic vessel maturation and valve formation. Blood 2013, 122, 598-607. [CrossRef]

28. Chen, H.; Brady Ridgway, J.; Sai, T.; Lai, J.; Warming, S.; Chen, H.; Roose-Girma, M.; Zhang, G.; Shou, W.; Yan, M. Context-dependent signaling defines roles of bmp9 and bmp10 in embryonic and postnatal development. Proc. Natl. Acad. Sci. USA 2013, 110, 11887-11892. [CrossRef]

29. Deleve, L.D.; Wang, X.; Hu, L.; McCuskey, M.K.; McCuskey, R.S. Rat liver sinusoidal endothelial cell phenotype is maintained by paracrine and autocrine regulation. Am. J. Physiol. Liver Physiol. 2004, 287, G757-G763. [CrossRef]

30. Géraud, C.; Schledzewski, K.; Demory, A.; Klein, D.; Kaus, M.; Peyre, F.; Sticht, C.; Evdokimov, K.; Lu, S.; Schmieder, A.; et al. Liver sinusoidal endothelium: A microenvironment-dependent differentiation program in rat including the novel junctional protein liver endothelial differentiation-associated protein-1. Hepatol. 2010, 52, 313-326. [CrossRef]

31. Deleve, L.D. Liver sinusoidal endothelial cells in hepatic fibrosis. Hepatology 2015, 61, 1740-1746. [CrossRef]

32. Xie, G.; Wang, X.; Wang, L.; Wang, L.; Atkinson, R.D.; Kanel, G.C.; Gaarde, W.A.; Deleve, L.D. Role of differentiation of liver sinusoidal endothelial cells in progression and regression of hepatic fibrosis in rats. Gastroenterology 2012, 142, 918-927. [CrossRef] [PubMed]

33. Korshunov, V.A.; Berk, B.C. Strain-dependent vascular remodeling: The "glagov phenomenon" is genetically determined. Circulation 2004, 110, 220-226. [CrossRef] [PubMed]

34. Rohan, R.M.; Fernández, A.; Udagawa, T.; Yuan, J.; D’Amato, R.J. Genetic heterogeneity of angiogenesis in mice. FASEB J. 2000, 14, 871-876. [CrossRef] [PubMed]

35. Gatti, D.; Maki, A.; Chesler, E.J.; Kirova, R.; Kosyk, O.; Lu, L.; Manly, K.F.; Qu, Y.; Williams, R.W.; Perkins, A.; et al. Genome-level analysis of genetic regulation of liver gene expression networks. Hepatology 2007, 46, 548-557. [CrossRef]

36. Steeland, S.; Timmermans, S.; Van Ryckeghem, S.; Hulpiau, P.; Saeys, Y.; Van Montagu, M.; Vandenbroucke, R.E.; Libert, C. Efficient analysis of mouse genome sequences reveal many nonsense variants. Proc. Natl. Acad. Sci. USA 2016, 113, 5670-5675. [CrossRef] [PubMed]

37. Stan, R.V.; Kubitza, M.; Palade, G.E. Pv-1 is a component of the fenestral and stomatal diaphragms in fenestrated endothelia. Proc. Natl. Acad. Sci. USA 1999, 96, 13203-13207. [CrossRef]

38. Herrnberger, L.; Hennig, R.; Kremer, W.; Hellerbrand, C.; Goepferich, A.; Kalbitzer, H.R.; Tamm, E.R. Formation of Fenestrae in Murine Liver Sinusoids Depends on Plasmalemma Vesicle-Associated Protein and Is Required for Lipoprotein Passage. PLoS ONE 2014, 9, e115005. [CrossRef]

39. Herrnberger, L.; Seitz, R.; Kuespert, S.; Bösl, M.R.; Fuchshofer, R.; Tamm, E.R. Lack of endothelial diaphragms in fenestrae and caveolae of mutant Plvap-deficient mice. Histochem. Cell Biol. 2012, 138, 709-724. [CrossRef]

40. Stan, R.V.; Tse, D.; Deharvengt, S.J.; Smits, N.C.; Xu, Y.; Luciano, M.R.; McGarry, C.L.; Buitendijk, M.; Nemani, K.V.; Elgueta, R.; et al. The diaphragms of fenestrated endothelia-Gatekeepers of vascular permeability and blood composition. Dev. Cell 2012, 23, 1203-1218. [CrossRef]

41. Schledzewski, K.; Géraud, C.; Arnold, B.; Wang, S.; Gröne, H.-J.; Kempf, T.; Wollert, K.C.; Straub, B.K.; Schirmacher, P.; Demory, A.; et al. Deficiency of liver sinusoidal scavenger receptors stabilin-1 and -2 in mice causes glomerulofibrotic nephropathy via impaired hepatic clearance of noxious blood factors. J. Clin. Investig. 2011, 121, 703-714. [CrossRef] [PubMed]

42. Herrera, B.; García-Álvaro, M.; Cruz, S.; Walsh, P.; Fernandez, M.; Roncero, C.; Fabregat, I.; Sanchez, A.; Inman, G.J. BMP9 Is a Proliferative and Survival Factor for Human Hepatocellular Carcinoma Cells. PLoS ONE 2013, 8, e69535. [CrossRef] [PubMed] 
43. Li, Q.; Gu, X.; Weng, H.; Ghafoory, S.; Liu, Y.; Feng, T.; Dzieran, J.; Li, L.; Ilkavets, I.; De Julio, M.K.; et al. Bone morphogenetic protein-9 induces epithelial to mesenchymal transition in hepatocellular carcinoma cells. Cancer Sci. 2013, 104, 398-408. [CrossRef] [PubMed]

44. Uehara, T.; Pogribny, I.P.; Rusyn, I. The DEN and CCl4-induced Mouse Model of Fibrosis and Inflammation-associated Hepatocellular Carcinoma. Curr. Protoc. Pharmacol. 2014, 66, 14-30. [PubMed]

45. Hammoutene, A.; Rautou, P.-E. Role of liver sinusoidal endothelial cells in non-alcoholic fatty liver disease. J. Hepatol. 2019, 70, 1278-1291. [CrossRef] [PubMed]

(C) 2019 by the authors. Licensee MDPI, Basel, Switzerland. This article is an open access article distributed under the terms and conditions of the Creative Commons Attribution (CC BY) license (http://creativecommons.org/licenses/by/4.0/). 